\title{
Bayesian approach for multi gamma radionuclide quantification applied on weakly attenuating nuclear waste drums
}

\author{
A. Clement, N. Saurel, G. Perrin and N. Gombert
}

\begin{abstract}
Gamma spectrometry is a passive non-destructive assay method used to quantify radionuclides present in nuclear objects. Basic methods using empirical calibration with a standard to quantify the activity of nuclear materials by determining the calibration coefficient are ineffective on non-reproducible nuclear objects such as waste packages. Package specifications such as composition or geometry change from one package to another and exhibit large variability of objects. The current standard quantification process uses numerical modelling of the measured scene with few available data such as geometry or composition, in particular density, material, screen, geometric shape, matrix composition, matrix and source distribution. Some of them are strongly dependent on package data knowledge and operator backgrounds. The French Atomic Energy Commission (CEA) is developing a methodology to quantify nuclear materials in waste packages and waste drums without operator adjustment and internal package configuration knowledge. This method suggests combining a stochastic approach which uses, among others, surrogate models available to simulate the gamma attenuation behaviour, a Bayesian approach considering conditional probability densities and prior information of problem inputs, and Markov Chain Monte Carlo algorithms (MCMC) which solve inverse problems, with gamma ray emission radionuclide spectra, and the outside dimensions of the objects of interest. The methodology has been tested to quantify actinide activity with a low bulk density matrix, weakly attenuating compositions, without information on the distribution of the source in terms of actinide masses and materials composing the drums. Activity uncertainties are taken into account.
\end{abstract}

Index Terms-Nuclear quantification, Bayes Theorem, MCMC, Monte Carlo sampling

\section{INTRODUCTION}

$\mathbf{T}$ HE quantification of nuclear materials is crucial in many branches of the nuclear industry such as nuclear power plants, in nuclear criticality safety or in nuclear decommissioning. Many nuclear detection techniques such as neutron detection methods and gamma spectrometry are carried out to accurately quantify radionuclide masses included in a large number of more or less complex objects. Some of the most widely used measurement systems for quantifying mass/activity of these materials are high-purity germanium (HPGe) gamma-ray detectors [1]. Compared to $\mathrm{NaI}$ detectors, the dominant feature of HPGe detectors is their

This work was supported by the Military Division of the French Atomic Energy Commision (CEA DAM).

A. Clement, N. Saurel and N. Gombert was with the Nuclear Measurement Laboratory, French Commissariat à l'Energie Atomique, CEA/DAM/VA, F21120 Is-sur-Tille (e-mails: alois.clement@cea.fr, nicolas.saurel@cea.fr).

G. Perrin was with the French Commissariat à l'Energie Atomique, CEA/DAM/DIF, F-91297 Arpajon CEDEX, France. excellent energy resolution around $1 \mathrm{keV}$ at $662 \mathrm{keV}$, which is a very usefull feature for identification and quantification of radionuclides such as ${ }^{239} \mathrm{Pu}$ or ${ }^{241} \mathrm{Am}$. A numerical method [2] has been developed to propose reliable and accurate characterization of HPGe detectors by combining 3D Monte Carlo particle transport simulation codes such as MCNP 6.2 [3] with applied mathematical tools. This numerical detector model is constructed in order to reduce global uncertainties of final quantification results by increasing the detection capability knowledge of HPGe detectors.

To identify and quantify gamma emitting nuclides included in different kind of objects, such as nuclear waste packages, one needs to calculate the activity $A$ by :

$$
A=\frac{S(E)}{\epsilon(E) t I_{\gamma}(E)},
$$

where:

- $E$ : Energy (MeV)

- $A$ : Activity of a radionuclide of interest (Bq)

- $S(E)$ : Net counting area of the full energy peak at the energy $E$ (counts)

- $I_{\gamma}(E)$ : Branching ratio of radionuclide at the energy $E$

- $t$ : Acquisition duration (s)

- $\epsilon(E)$ : Absolute efficiency coefficient at the energy $E$, also called attenuation law

which allows the mass $m$ of the radionuclide of interest to be calculated with:

$$
m=\frac{A}{A_{m}}
$$

where:

- $m$ : Mass of the radionuclide of interest included in the measured object $(\mathrm{g})$

- $A$ : Activity of the radionuclide of interest $(\mathrm{Bq})$

- $A_{m}$ : Specific activity of the radionuclide of interest $(\mathrm{Bq} / \mathrm{g})$

The $\epsilon(E)$ coefficient is related to the capability of a measured object to reduce the gamma signal coming from a nuclear material of interest. It depends on object features such as internal layout, screens, density, position of the gamma source relative to the HPGe detector, internal materials and energy. Though the $S(E)$ spectrum net extracted areas 
can be easily determined with accuracy [5], calculation of $\epsilon(E)$ is a difficult process for complex objects in terms of internal layout and composition such as nuclear waste drums [2]. Current and common calculation methods use Monte Carlo simulation codes as MCNP6 [3] to model measured scenes and approach as precisely as possible the real values of the $\epsilon(E)$ coefficient. Several years ago, new kinds of nuclear numerical quantification methods dealing with applied mathematics and stochastic approaches [6] proposed emulating outcomes of interest such as $\epsilon(E)$ to solve the inverse problem of quantification and to estimate the mass distribution of a radionuclide of interest included in objects such as nuclear waste drums. In this way, Carasco [7] proposes to couple gamma ray spectrometry and tomography in a Bayesian framework to characterize radionuclides of interest such as ${ }^{239} \mathrm{Pu}$ in nuclear waste. Moreover, Laloy et al. [8] introduce a Bayesian approach to characterise activities of radioactive waste from Segmented Gamma Scanning (SGS).

Section II presents a new approach to the mass quantification problem by considering data of interest as random variables using an MCNP-based surrogate for Bayesian inversion through an MCMC algorithm. Section III gives experimental results considering a measurement database of nuclear waste drums. Section IV provides discussions and conclusions.

\section{BAYESIAN THEORY APPLIED TO GAMMA SPECTROMETRY}

\section{A. Statistical quantification approach}

The quantification process that is considered here aims to estimate the probability density function (PDF) of a radionuclide of interest of mass $m$. Let us suppose the radionuclide of interest is a multi gamma-emitter. Via specific software for gamma spectrum analysis [9] [10], we obtain net extracted areas from a measured gamma spectrum. Let us assume there are $N$ net extracted areas gathered in vector $\mathbf{S}=\left\{S\left(E_{n}\right), n \in[1, N]\right\}$, relative to $\left(E_{n}\right)_{n \in[1, N]}$ energies of the multi gamma-emitter radionuclide, and $\mathbf{X} \in \Xi_{D}$ represents a $D$-dimension vector of inputs needed for the calculation of the absolute efficiency calibration coefficients vector $\epsilon(\mathbf{X})=\left\{\epsilon_{n}(\mathbf{X}), n \in[1, N]\right\}$ estimation.The $\mathbf{X}$ vector is composed of variables impacting the detection efficiency: measurement distance, cylindrical dimensions (radius, height), proportion of materials composing the cylinder (vinyl, iron, plutonium), bulk density, etc. Details are available in [6].

Hence, (1) and (2) lead to the following equation:

$$
\forall n \in[1, N], \quad S\left(E_{n}\right)=A \epsilon_{n}(\mathbf{X}) I_{\gamma}^{n} t=m A_{m} \epsilon_{n}(\mathbf{X}) I_{\gamma}^{n} t
$$

Let us suppose that $\mathbf{Y}^{\mathbf{o b s}}$ is the observation vector with $\mathbf{I}_{\gamma}$, $t$ and $A_{m}$ deterministic and known, defined as:

$$
\mathbf{Y}^{\mathbf{o b s}}=\frac{\mathbf{S}}{\mathbf{I}_{\gamma} t A_{m}}+\xi=m \epsilon(\mathbf{X})+\xi, \quad \xi \sim \mathcal{N}\left(0, \sigma_{\text {obs }}\right) .
$$

In (4), $\xi$ represents the $\mathbf{Y}^{\text {obs }}$ observation uncertainty vector. Let us assume that all of its coefficients are related to normal distributions centered at zero with $\sigma_{\text {obs }}=\left(\sigma_{o b s, n}\right)_{n \in[1, N]}$ as standard deviation vector. This condition is verified with a sufficient number of counts (central limit theorem).

In considering $\mathbf{Y}^{\mathbf{o b s}}, m, \epsilon(\mathbf{X})$, and $\mathbf{X}$ as random variables, or vectors composed of random variables, the marginal PDF for $m$ mass given $\mathbf{Y}^{\mathbf{o b s}}$, expressed as $f\left(m \mid \mathbf{Y}^{\mathbf{o b s}}\right)$, is given by Bayes theorem written in terms of PDFs [11]. The probability density $f\left(m \mid \mathbf{Y}^{\mathbf{o b s}}\right)$ depends on joint PDF $f\left(m, \mathbf{Y}^{\mathbf{o b s}}\right)$ and $f_{\mathbf{Y}^{\text {obs }}}\left(\mathbf{Y}^{\mathbf{o b s}}\right)$ the marginal PDF of $\mathbf{Y}^{\text {obs }}$ [2], as expressed in the following equation:

$$
f\left(m \mid \mathbf{Y}^{\mathbf{o b s}}\right)=\frac{f\left(m, \mathbf{Y}^{\mathbf{o b s}}\right)}{f_{\mathbf{Y}} \mathbf{\text { obs }}\left(\mathbf{Y}^{\mathbf{o b s}}\right)} \propto f\left(\mathbf{Y}^{\mathbf{o b s}} \mid m\right) \pi(m) .
$$

The distribution $\pi(m)$ is called prior distribution [11] and is typically based on hypotheses, experience, or subjective opinion about $m$ mass. Taking dependencies upon $\mathbf{X}$ into account leads to (6):

$$
f\left(m \mid \mathbf{Y}^{\mathbf{o b s}}\right) \propto \int_{\mathbf{X}} f\left(\mathbf{Y}^{\mathbf{o b s}} \mid m, \mathbf{X}\right) \pi(m \mid \mathbf{X}) \pi(\mathbf{X}) d \mathbf{X}
$$

\section{B. Hypotheses}

The objective is to obtain an estimation of $f\left(m \mid \mathbf{Y}^{\mathbf{o b s}}\right)$, the probability density of $m$ mass given the $\mathbf{Y}^{\text {obs }}$ vector. Let us assume some hypotheses about necessary PDFs to provide the mass distribution:

- $\mathbf{Y}^{\mathbf{o b s}}$ is a vector composed of $N$ independent random variables $Y_{n}^{o b s}$ related to normal distributions:

$$
\forall n \in[1, N], Y_{n}^{o b s} \mid m \sim \mathcal{N}\left(\mu_{n}(m, \mathbf{X}), \sigma_{n}(m, \mathbf{X})^{2}\right),
$$

with $\mu_{n}(m, \mathbf{X})$ and $\sigma_{n}(m, \mathbf{X})$ respectively the mean and standard variation ;

- Let us assume there is a priori no dependence between $m$ and $\mathbf{X}$, and between the components of $\mathbf{X}$. Hence, the efficiency $\epsilon(\mathbf{X})$ does not depend on the $m$ radionuclide mass. In this case, the prior distribution $\mathbf{X}=\left(X_{d}\right)_{d \in[1, D]}$ can be expressed as:

$$
\pi(m \mid \mathbf{X}) \pi(\mathbf{X})=\pi(m) \pi(\mathbf{X})=\pi(m) \prod_{d=1}^{D} \pi\left(X_{d}\right)
$$

- Each prior distribution provides information about its own random variable [11]. A well-known variable is associated with tight normal prior distribution $\mathcal{N}\left(\mu_{0}, \sigma_{0}\right)$, and thus it provides information about variable behaviour. A variable with no real information, such as $m$ mass or $\rho$ density of the object, is associated with uniform prior distribution $\mathcal{U}\left(x_{\min }, x_{\max }\right)$. This method makes it possible to control real knowledge about the measured object and test different hypotheses on unknown variables.

\section{MCMC sampling and surrogate models}

The calculation of the conditional PDF of $\mathrm{m} f\left(\mathrm{~m} \mid \mathbf{Y}^{\mathbf{o b s}}\right)$ leads to the evaluation of the integral over $\mathbf{X}$ appearing in (6). When the dimension of $\mathbf{X}$ is relatively high, directly computing the integral given by (6) is hardly feasible. A solution 
to get around the problem is to sample $m$ mass conditional PDF by using Markov Chain Monte Carlo (MCMC) methods [12] [13]. The Metropolis-Hastings algorithm allows us to sample the mass $m$ and $\mathbf{X}$ components PDFs by using Markov Chain theory [14]. This method typically needs several tens of thousands of calls to the simulation code providing evaluations of the forward model provided by (4), in which the efficiency should be evaluated by a Monte Carlo particle transport simulation, e.g. with MCNP. Because of the computational intractibility to rapidly evaluate $\epsilon(\mathbf{X})$ directly with the particle transport code MCNP6, a Kriging surrogate model [15] [16] [17] is proposed to emulate it. A surrogate model [18] requires a limited number of calls to the code, which are gathered in a design of experiments (DoE), in order to first accurately and quickly build an outcome of interest function $M$, and finally predict new values of interest such as:

$$
\forall \mathbf{X} \in \Xi_{D}, \quad \epsilon(\mathbf{X}) \approx M(\mathbf{X})
$$

A practical and well-known DoE building technique is Latin Hypercube Sampling (LHS) [19] [20]. This kind of DoE gives very interesting space filling properties by dealing with different criterion maximization such as the minimax criterion [21]. The $M$ model construction required 500 experiments with MCNP and around 16 hours of total computation time with 72 processing cores (Intel®Xeon(R) CPU E5-2699 v3 @ $2.3 \mathrm{GHz} \times 16$ (x2)). The Kriging computation time was about 1 second (1 core). It was built with DiceKriging and DiceOptim libraries [15] using the $\mathrm{R}$ programming language [22]. The surrogate is built with the Matern 5/2 covariance function without nugget effect.

\section{Calculation of the $f\left(m \mid \mathbf{Y}^{\mathbf{o b s}}\right)$ conditional probability den-} sity

The objective is to obtain $m$ mass conditional PDF $f\left(m \mid \mathbf{Y}^{\mathbf{o b s}}\right)$. Considering (6-8-9) and the given hypotheses detailed below, $f\left(\mathbf{Y}^{\mathbf{o b s}} \mid m\right)$ can be written as :

$$
f\left(\mathbf{Y}^{\mathbf{o b s}} \mid m\right)=\prod_{n=1}^{N} f\left(Y_{n}^{o b s} \mid m\right)=\prod_{n=1}^{N} \frac{1}{\sqrt{2 \pi \sigma_{n}}} e^{-\frac{\left(\mu_{n}^{o b s}-\mu_{n}\right)^{2}}{2 \sigma_{n}^{2}}}
$$

where $\left(\mu_{n}\right)_{n \in[1, N]}$ and $\left(\sigma_{n}\right)_{n \in[1, N]}$ are determined by considering the uncertainty of the net extracted area vector $\mathbf{S}$ from (4). Here, $\left(\mu_{n}^{o b s}\right)_{n \in[1, N]}$ vector represents observation inputs coming from the areas extracted from the gamma spectrum.

Due to its calculation process, which uses the Kriging emulation method presented above, the $\epsilon(\mathbf{X})$ random variable vector can be written as following:

$$
\begin{gathered}
\epsilon(\mathbf{X})=\nu^{M}(\mathbf{X})+\sigma^{M}(\mathbf{X}) \delta_{\sigma}+e_{\text {model }}(\mathbf{X}) \delta_{e} \\
\delta_{\sigma} \sim \mathcal{N}\left(0, \mathbf{1}_{\mathbf{N}}\right), \quad \delta_{e} \sim \mathcal{N}\left(0, \sigma_{\text {model }}^{\mathbf{2}}\right),
\end{gathered}
$$

with $\nu^{M}(\mathbf{X})=\left(\nu_{n}^{M}(\mathbf{X})\right)_{n \in[1, N]}$ the predictive mean vector of $\epsilon(\mathbf{X})$ and $\sigma^{M}(\mathbf{X})=\left(\sigma_{n}^{M}(\mathbf{X})\right)_{n \in[1, N]}$ its standard deviation vector coming from the $M$ Kriging surrogate model from
(9). $e_{\text {model }}(\mathbf{X})$ represents the model error. It should be noted that this uncertainty is assumed to be insignificant but it is retained in the calculation process. It should also be noted that uncertainties on the necessary MCNP6 values for Kriging model construction are taken into account on a point by point basis. Consequently, this error does not appear in (11).

Hence, (4-11) let us express $\mathbf{Y}^{\text {obs }}$ PDF from $Y_{n}^{o b s}$ PDFs:

$$
\begin{gathered}
\forall n \in[1, N], \quad Y_{n}^{o b s} \mid m \sim \mathcal{N}\left(\mu_{n}, \sigma_{n}^{2}\right), \\
\mu_{n}=m \nu_{n}^{M}(\mathbf{X}), \\
\sigma_{n}^{2}=m^{2}\left(\sigma_{n}^{M}(\mathbf{X})^{2}+\sigma_{\text {model }}^{2}\right)+\sigma_{o b s, n}^{2} .
\end{gathered}
$$

To finish, considering (6-8-11-12-13), the $m$ mass conditional PDF can be expressed as follows:

$f\left(m \mid \mathbf{Y}^{\mathbf{o b s}}\right) \propto \int_{\mathbf{X}} \prod_{n=1}^{N} \frac{1}{\sqrt{2 \pi \sigma_{n}}} e^{-\frac{\left(\mu_{n}^{o b s}-\mu_{n}\right)^{2}}{2 \sigma_{n}^{2}}} \pi(m) \prod_{d=1}^{D} \pi\left(X_{d}\right) d_{\mathbf{X}}$

The desired $m$ mass conditional PDF may also be estimated by Metropolis-Hastings algorithm as explained below.

\section{E. About the $\xi$ random variable}

Henceforth, the aim is to consider $m$ mass PDF and to sample it with MCMC methods such as the MetropolisHastings algorithm. Nevertheless, a remaining variable has to be approached. Because of the difficulty to accurately quantify the $\sigma_{\text {obs }}$ standard deviation vector appearing in (4) and depending on the spectrum area extraction method, two hypotheses $\left(H_{1}\right.$ and $\left.H_{2}\right)$ about it are possible :

$$
\begin{array}{llrl}
H_{1}: & \xi \sim \mathcal{N}\left(0, \sigma_{\text {obs }}=\alpha \mathbf{Y}_{\text {obs }}\right), & \alpha \in[0,1], \\
H_{2}: & \xi \sim \mathcal{N}\left(0, \sigma_{\text {obs }}=\sigma \mathbf{1}_{\mathbf{N}}\right), & \sigma \in \mathbb{R} .
\end{array}
$$

The first hypothesis $H_{1}$ proposes making the $\sigma_{o b s}$ vector proportional to observable values (15). In the second hypothesis $H_{2}, \sigma_{o b s}$ is constant and equal to an arbitrary value $\sigma$ for all $N$ components (16).

\section{EXPERIMENTAL RESULTS}

\section{A. Hypotheses}

The stochastic quantification method has been coded with Python3.7 [23]. The MCMC algorithm is a Random Walk Metropolis-Hastings algorithm. [13] A custom implementation was entirely coded by ourselves without an existing package. The first hypothesis $H_{1}(15)$ is considered with $\alpha=0.05$. All of the net extracted area uncertainties considered here are less than $5 \%$. This uncertainty was also fixed to $5 \%$ to simplify the calculations. To test the proposed Bayesian approach of multi gamma-emitter radionuclide quantification, a nuclear waste drum measurements database was used. This database comes from MADAGASCAR, a Segmented Gamma Scanning [24] [25] nuclear measurement system used by the CEA to identify and quantify radionuclide activities in waste drums before sending them to appropriate outlets. The technical features of 
the drums to be considered by the MADAGASCAR system are :

1) Detection limit about $1 \mathrm{MBq}$ with maximum accepted activity about $200 \mathrm{GBq}$

2) Relative bulk density of drums in $[0,0.4]$

3) 100 and 200 litre nuclear waste drum type

4) Fixed measuring distance

5) The standard deviations of measurements is set at \pm $42 \%(2 \sigma)$

The standard deviations about $\pm 42 \%$ is a standard value provided by a global validation study of MADAGASCAR. The system uses a radioactive source to obtain transmission coefficients of drums and to evaluate the standard calibration efficiency. The MADAGASCAR quantification method is COFRAC [26] accredited.

The multi gamma-emitter radionuclide of interest to be quantified is ${ }^{239} \mathrm{Pu}$. Here, the objective is to test the capabilities of the stochastic method by comparing quantification results of ${ }^{239} \mathrm{Pu}$ activities between the described Bayesian approach and the MADAGASCAR system quantification results.

To do so, a database of 242 measurements obtained by MADAGASCAR in 2017 on 100 litre drums was used. The HPGe detector of MADAGASCAR was a Broad Energy Germanium detector (BEGe) sold by Canberra (BE2820). The distribution and the form of the ${ }^{239} \mathrm{Pu}$ masses in drums are unknown. Moreover, the method ignores the materials composing the drums of the database. The drums are composed of lightweight materials (vinyl plastics, papers, PVC, etc.) and heavier materials such as metals (iron, steel, aluminium, etc.).

Each MADAGASCAR measurement considered in the base leads to :

1) $A_{M}^{P u^{239}}:$ MADAGASCAR declared activity of ${ }^{239} \mathrm{Pu}$

2) Gross and net weights of 100 litre drums

Moreover, each measurement of the database has its own acquisition gamma spectrum derived from MADAGASCAR. Also, the input data of the stochastic method are :

1) $S_{i}^{239} P u:$ Net extracted areas of the $i^{t h}{ }^{239} P u$ energies of interest

2) Acquisition duration

3) Measurement distances

4) Drum volume (100 litres) and dimensions (radius and height)

5) Bulk density

The ${ }^{239} \mathrm{Pu}$ energies of interest are 94.66, 129.29, 203.55, $345.01,375.05$ and $413.71 \mathrm{keV}$. Peaks that are absent are ignored by the calculation procedure. The measurement distance and drum dimensions are identical for all elements of the database, respectively $58.4 \mathrm{~cm}$ and $23 \mathrm{~cm}$ of radius and $68 \mathrm{~cm}$ of height. These data are used by the method as a priori objective, i.e. related to Gaussian prior probability densities with known variances (II-B).

Concerning the efficiency response of the germanium detector, this is obtained by modelling with a Kriging surrogate model. The design of experiments used is similar to that built for modelling the efficiency of the measurement scene (LHS). The MCNP6 calculation code is used to build a digital twin of the detector via the use of As Built plans of the detector provided by the manufacturer.

\section{B. About statistics}

The convergence process of the MCMC chains is investigated visually in Fig. 1.

The experimental validation process is based on the following objectives :

1) Repeatability, reproducibility

2) Linearity - Validation

3) Coverage

4) Comparison

Each item is definied in Table I.

The statistical estimators used to evaluate the stochastic method quantification results and to compare to the MADAGASCAR measurement results are :

1) The mode of the ${ }^{239} \mathrm{Pu}$ estimated mass probability distribution obtained via a log-normal fit : the data obtained by the stochastic method represent the posterior probability density functions of ${ }^{239} \mathrm{Pu}$ masses, the mode estimator of such distributions therefore represents the most probable mass proposed by the stochastic method. The capability of the fit to properly describe the probability density is assessed using Quantile-Quantile plot (QQ-plot), Probability-Probability plot (PP-plot) and by comparing the empirical and theoretical Cumulative Density Functions (CDF).

2) The $2.5 \%$ and $97.5 \%$ quantile values, leading to the $95 \%$ confidence interval : these two estimators were chosen due to the skewness of the probability distributions obtained by the stochastic method.

The effective posterior samples considered for the log-normal fit were chosen in getting $1 \%$ of the estimated posterior PDF, composed by the 35 independent MCMC chains. These samples are considered to be independent after appropriate thinning (one sample every hundred). The burn-in of the MCMC chains have been removed. The MCMC algorithm was tweaked to have an acceptance rate around 30\% [14]leading to around 15000 iterations per chain. The number of effective posterior samples forming the estimated posterior PDF is about 5000 .

\section{Repeatability, reproducibility}

This part proposes checking the criterion of reproducibility (repeatability) of the stochastic quantification method. The 

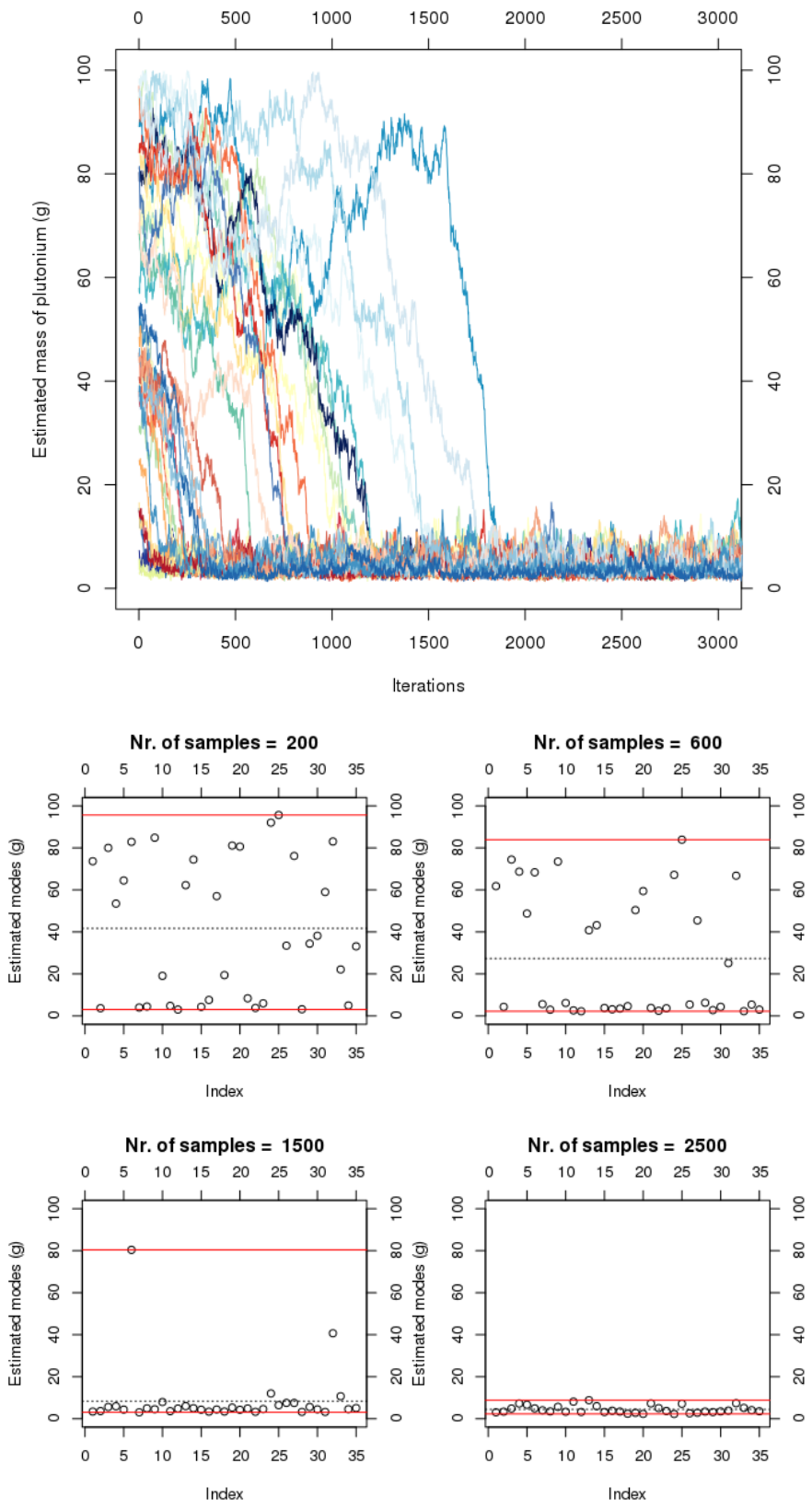

Fig. 1. Convergence visualization. On the top : the $35 \mathrm{MCMC}$ chains of 1 of the 242 measurements of the MADAGASCAR system. On the bottom : the estimated mass of plutonium of the $35 \mathrm{MCMC}$ chains for four different values of the MCMC iteration. In red, the maximum and minimum values of the estimated mass of plutonium. In dotted, the mean of the 35 estimated mass of plutonium.

objective is to quantify the standard deviation associated with the use of the described stochastic method. This involves using the method on the same objects several times in order to observe estimator variations.

In order to statistically cover the domain of definition of standard deviations of the estimators sought, the method is repeated at least 35 times ( $>30$ for normal law conditions) on the 10 randomly selected items. The calculation time is set at
TABLE I

EXPERIMENTAL STATISTICAL CRITERIA

\begin{tabular}{|l|l|l|}
\hline Criteria & Methodology & Data \\
\hline $\begin{array}{l}\text { Repeatability, } \\
\text { reproducibility }\end{array}$ & $\begin{array}{l}\text { Repeat the method for 10 ram- } \\
\text { domly selected cases and anal- } \\
\text { yse standard deviations (1 } \sigma \text { ) of } \\
\text { the mode estimator. }\end{array}$ & $\begin{array}{l}\text { MADAGASCAR } \\
\text { database (10 items). }\end{array}$ \\
\hline $\begin{array}{l}\text { Linearity } \\
\text { Validation }\end{array}$ & $\begin{array}{l}\text { 239 Pu mass PDF evaluation } \\
\text { of a set of standard sources } \\
\text { without matrix effect (empty } \\
\text { drums). Calculation of 68\%CI } \\
\text { of mass PDF. }\end{array}$ & $\begin{array}{l}239 P u \text { standard sources } \\
(6 \text { items) }\end{array}$ \\
\hline Coverage & $\begin{array}{l}\text { Mass PDF recovery assess- } \\
\text { ment obtained by the stochas- } \\
\text { tic method with the 95\%CI re- } \\
\text { turned in by MADAGASCAR. }\end{array}$ & $\begin{array}{l}\text { MADAGASCAR } \\
\text { database (242 items) }\end{array}$ \\
\hline Comparison & $\begin{array}{l}\text { 239 Pu PDF mass evaluation } \\
\text { and verification of the presence } \\
\text { of MADAGASCAR results in } \\
\text { estimated PDF. }\end{array}$ & $\begin{array}{l}\text { MADAGASCAR } \\
\text { database (242 items) }\end{array}$ \\
\hline
\end{tabular}

PDF $=$ Probability Density Function, $68 \% \mathrm{CI}=68 \%$ Confidence Interval, $95 \% \mathrm{CI}=95 \%$ Confidence Interval

$15 \mathrm{~min}$ per sample and per measurement, i.e. $8 \mathrm{~h} 45 \mathrm{~min}$ for each item (35 samples). The results are summarized in Table II.

TABLE II

REPRODUCTIBILITY AND REPEATABILITY RESULTS

\begin{tabular}{|l|c|c|c|c|c|c|}
\hline Item & $\bar{M}_{M o d}(\mathrm{~g})$ & $S D_{M o d}(\%)$ & $\bar{M}_{Q 1}(\mathrm{~g})$ & $S D_{Q 1}(\%)$ & $\bar{M}_{Q 2}(\mathrm{~g})$ & $S D_{Q 2}(\%)$ \\
\hline 1. & 3.22 & 3.16 & 1.89 & 1.87 & 7.85 & 12.1 \\
\hline 2. & 2.45 & 5.05 & 1.32 & 3.30 & 5.73 & 6.98 \\
\hline 3. & 4.73 & 7.08 & 2.71 & 3.38 & 12.1 & 22.1 \\
\hline 4. & 1.01 & 1.10 & 0.59 & 0.88 & 2.28 & 2.72 \\
\hline 5. & 0.63 & 0.97 & 0.37 & 0.79 & 1.37 & 1.80 \\
\hline 6. & 4.92 & 10.0 & 2.82 & 4.07 & 13.1 & 28.1 \\
\hline 7. & 2.39 & 2.31 & 1.42 & 1.69 & 5.30 & 5.47 \\
\hline 8. & 3.48 & 6.76 & 1.96 & 2.00 & 10.2 & 25.9 \\
\hline 9. & 1.41 & 1.96 & 0.80 & 1.19 & 3.40 & 6.02 \\
\hline 10. & 0.59 & 1.82 & 0.30 & 0.71 & 1.60 & 3.46 \\
\hline
\end{tabular}

$\bar{M}_{M o d}=$ Mean of modes, $S D_{M o d}=$ Relative standard deviation of modes, $\bar{M}_{Q 1}=$ Mean of quantiles at $2.5 \%, S D_{Q 1}=$ Relative standard deviation of quantiles at $2.5 \%, \bar{M}_{Q 2}=$ Mean of quantiles at $97.5 \%, S D_{Q 2}=$ Relative standard deviation of quantiles at $97.5 \%$

Fig. 2 shows the results of one of the ten measurements of the reproductibility study. The mean and standard deviation of the standard deviations of the mode estimator are respectively $4.02 \%$ and $3.06 \%$. Fig. 3 represents the standard deviations of the three estimators tested as a function of the mode mass of plutonium estimated by the stochastic method.

Fig. 3 shows a strong dependence of tested estimator standard deviations on the mean of the estimated mode masses. These standard deviations increase as the mean of the estimated mode mass increases. The standard devation of the estimated mean mode mass is less than or equal to $10 \%$ for all 10 tested measurement cases. The standard deviations of 


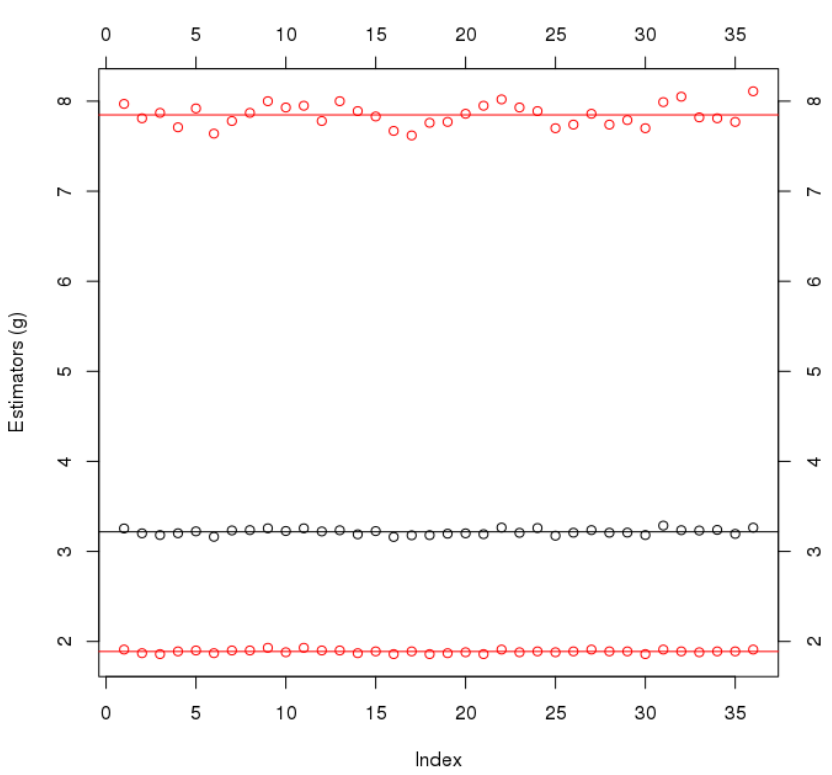

Fig. 2. Example of reproducibility study results for one of the ten measurements. Black circles represent estimated modes. Red circles represent estimated quantiles of $2.5 \%$ and $97.5 \%$. Lines represent means of each estimator.

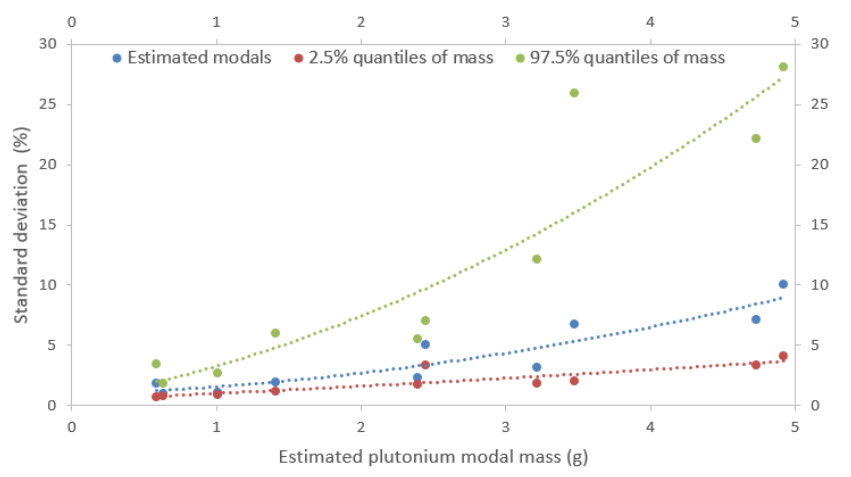

Fig. 3. Standard deviations of mode and $2.5 \%$ and $97.5 \%$ quantile estimators as a function of the mean of the estimated mode masses (Table II). Dotted curves represent second order polynomial fits of each of the three sets of data.

the quantile estimators vary from a few percent to almost $30 \%$. Attempts were made with a computation time of $15 \mathrm{~min}$. An increase in the computation time (approximately one hour) would significantly reduce the standard deviations of the three tested estimators. This study makes it possible to analyze the behavior of the variances of the three estimators. On one hand, the results allow to have a great confidence in two estimators (modal and quantile at 2.5\%). On the other hand, the variance of the quantile at $97.5 \%$ must be considered with care.

\section{Linearity - Validation}

This section aims to check the linearity criterion of the stochastic method as defined in Table I and to validate the method. To do this, 6 measurements of plutonium standard sources placed in empty drums were used. These measurements were carried out on the MADAGASCAR

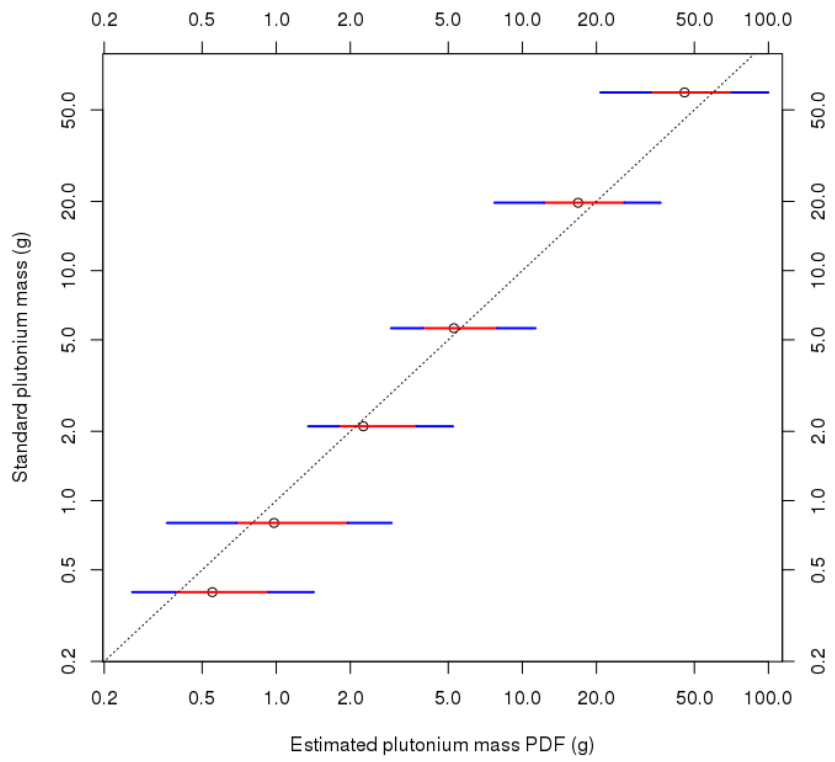

Fig. 4. Linearity - validation study results of the stochastic method applied to six plutonium standard masses from 0 to $60 \mathrm{~g}$. Red and blue segments represent respectively the $68 \%$ and $95 \%$ CI of estimated PDF of the total plutonium mass by the method. The circle on each segment represents the estimated mode.

system validation process. The total plutonium masses of standard sources are : $0.400,0.800,2.104,5.619,19.731$ and 59.541 grams. The isotopy of plutonium is taken into account. The six energies of interest of the ${ }^{239} \mathrm{Pu}$ used in the stochastic method are unchanged [III-A]. For each of the plutonium standard sources, the stochastic method processes for 60 min with 35 cores about of computing power. We used the same CPU as in Section. II-C. The calculation time of one hour makes it possible to limit the effects on the standard deviation of the estimators observed in the reproducibility study.Fig. 4 and Table III summarize the linearity study results.

TABLE III

LINEARITY AND VALIDATION RESULTS

\begin{tabular}{|l|c|c|c|c|c|c|}
\hline Item & $M_{P u}(\mathrm{~g})$ & $M_{Q 1}(\mathrm{~g})$ & $M_{Q 2}(\mathrm{~g})$ & $M_{M o d}(\mathrm{~g})$ & $M_{Q 3}(\mathrm{~g})$ & $M_{Q 4}(\mathrm{~g})$ \\
\hline 1. & 0.400 & 0.26 & 0.39 & 0.55 & 0.93 & 1.42 \\
\hline 2. & 0.800 & 0.36 & 0.69 & 0.98 & 1.95 & 2.94 \\
\hline 3. & 2.104 & 1.35 & 1.79 & 2.26 & 3.72 & 5.22 \\
\hline 4. & 5.619 & 2.93 & 3.94 & 5.27 & 7.91 & 11.3 \\
\hline 5. & 19.731 & 7.71 & 12.2 & 16.9 & 26.0 & 36.4 \\
\hline 6. & 59.541 & 20.8 & 33.3 & 45.6 & 71.0 & 99.8 \\
\hline
\end{tabular}

$M_{P u}=$ Plutonium total mass of standard source, $M_{Q 1}=$ Estimated plutonium mass at $2.5 \%$ quantile, $M_{Q 2}=$ Estimated plutonium mass at $16 \%$ quantile, $M_{M o d}=$ Estimated mode of plutonium mass, $M_{Q 3}=$ Estimated plutonium mass at $84 \%$ quantile, $M_{Q 4}=$ Estimated plutonium mass at $97.5 \%$ quantile

For all six measurement cases, the total plutonium mass is included in the $68 \%$ confidence interval $(68 \% \mathrm{CI})$ of the estimated mass PDF, i.e. in the interval bounded by the $16 \%$ 
and $84 \%$ quantiles. This is similar to $1 \sigma$ interval of a normal distribution. Since the drums are empty, the effect tested is the self-absorption of plutonium. This effect increases as the amount of plutonium increases. The results show the capacity of the stochastic method to take this effect into account for relatively large quantities of plutonium. This study also makes it possible to validate the method on standards.

\section{E. Coverage}

The purpose of this section is to verify the conformity of the coverage criterion as defined in Table I. To do so, the 242 measurements of the MADAGASCAR database are used throughout the stochastic method. For each measurement, the ${ }^{239} \mathrm{Pu}$ net extracted areas of energies of interest are used throughout the stochastic method. The three estimators proposed in III-B are used for each item. As a reminder, these are the mode of the estimated probability distribution and quantiles at $2.5 \%$ and $97.5 \%$. A reduced mass is obtained for each of the 242 samples by dividing the three estimators tested by the mass of ${ }^{239} \mathrm{Pu}$ returned by the MADAGASCAR system. We have:

$$
M_{R e d}^{i}=\frac{M_{M o d}^{i}}{M_{M A D A}^{i}}
$$

Note that for item $\# i, M_{\text {Red }}^{i}$ represents the reduced plutonium mass, $M_{M o d}$ is the estimated mode plutonim mass of the stochastic method and $M_{M A D A}^{i}$ is the MADAGASCAR estimated plutonium mass. Fig. 5 summarizes the coverage study results. A recovery rate $R^{i}$ is calculated for all items in the database. This rate represents the capability of the $95 \%$ CI of the estimated conditional PDF to cover the $95 \% \mathrm{CI}$ of MADAGASCAR results, i.e. $\pm 42 \%$ [III-A]. This rate is defined as follows:

$$
R^{i}= \begin{cases}\min \left(\frac{q_{97.5}^{i}-0.58 M_{M A D A}^{i}}{0.84 M_{M A D A}^{i}}, 1\right), & \text { if } q_{97.5}^{i} \geq 0.58 M_{M A D A}^{i} \\ \min \left(\frac{1.42 M_{M A D A}^{i}-q_{2.5}^{i}}{0.84 M_{M A D A}^{i}}, 1\right), & \text { if } q_{2.5}^{i} \leq 1.42 M_{M A D A}^{i} \\ 0, & \text { otherwise }\end{cases}
$$

Note that for item $\# i, q_{2.5}^{i}$ and $q_{97.5}^{i}$ represent respectively the $2.5 \%$ and $97.5 \%$ quantiles of the estimated posterior PDF. The calculation results indicate that $92.3 \%$ of the tested measurements have a recovery rate greater than 0.5 . In other words, in more than 9 out of 10 cases, the $95 \%$ CI returned by the stochastic method represents at least $50 \%$ of that returned by MADAGASCAR system. The results are presented in Fig. 6.

The results presented in Fig. 5 show a good response of the stochastic method between 0 and $2 \mathrm{~g}$ of plutonium. The mode estimator is consistent and correctly represents the mass of plutonium obtained on the MADAGASCAR system. Nevertheless, an average positive bias about $25 \%$ appears for increasing mode values of estimated plutonium mass.

\section{F. Comparison}

The purpose of this part is to check the comparison criterion as defined in Table I. To do so, the 242 measurements of the
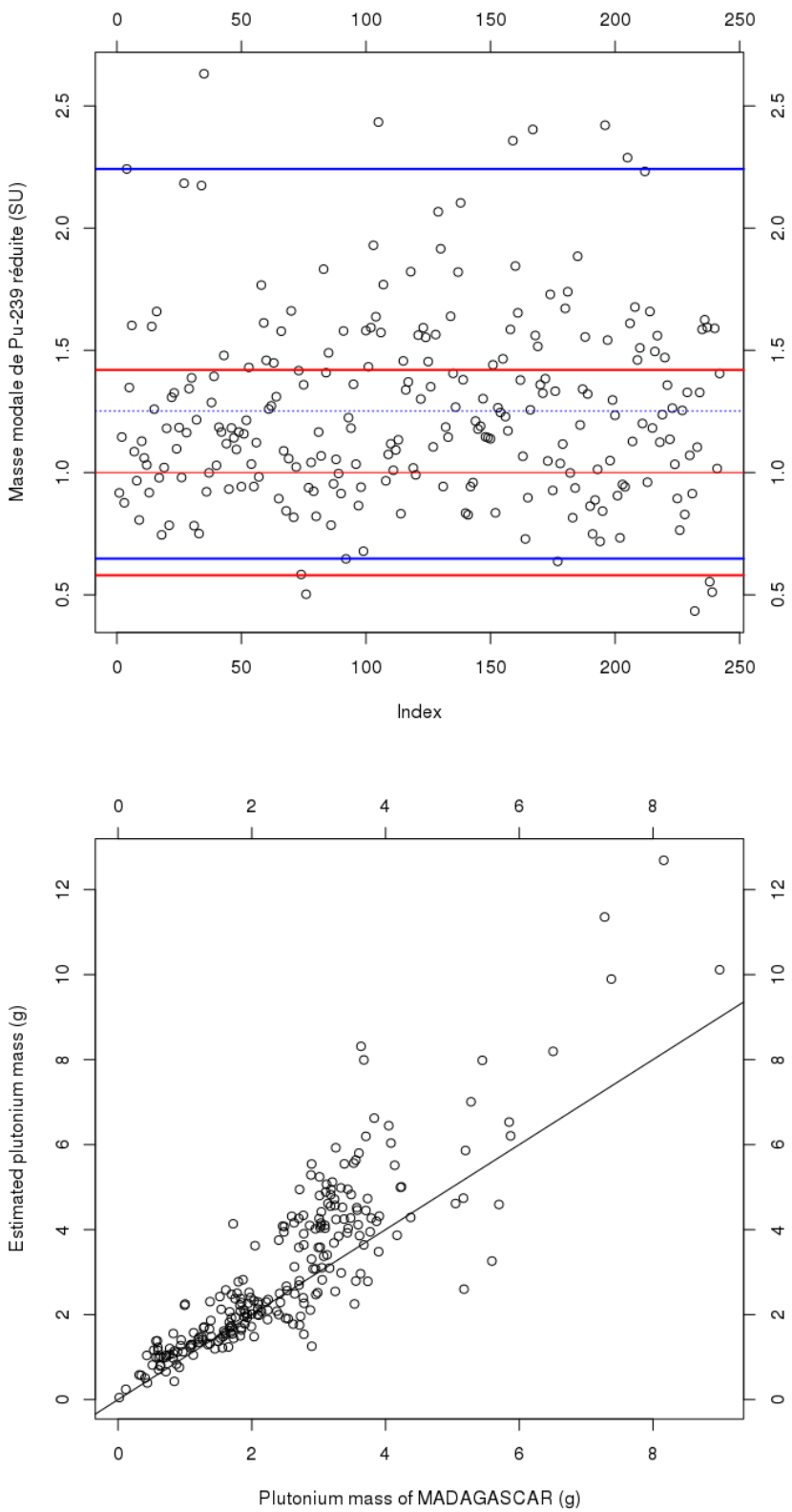

Fig. 5. Coverage study results. 1 - On top : Representation of the reduced plutonium mass for the 242 measurements. Solid blue lines represent limits of the $95 \%$ CI reported by the stochastic method for all items from the database; the dotted blue line represents the average of estimated modes. Solid red lines represent the $95 \%$ CI limits of all items from the database. 2 - On the bottom : Representation of the estimated mode mass in function of the estimated MADAGASCAR mass for all items from the database.

MADAGASCAR database are used. For each measurement, the mass mode is estimated by the stochastic method and we check its presence in the 95\% CI of MADAGASCAR mass results. We obtain that $91 \%$ of mass modes estimated by the stochastic method are included in the 95\% CI of MADAGASCAR mass results, so more than 9 out of 10 cases.

\section{DISCUSSIONS AND CONCLUSIONS}

The results obtained through the study of criteria as defined in Table I make it possible to estimate the PDF of plutonium 


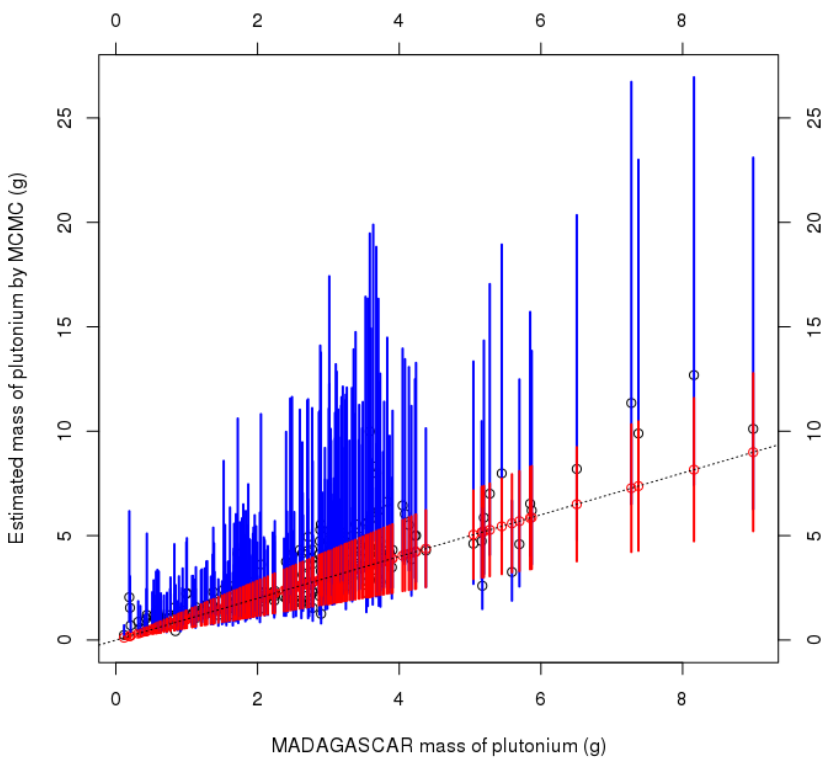

Fig. 6. Recovery rate results. In blue, the estimated conditional PDF of the plutonium masses $(95 \% \mathrm{CI})$. In red, the standard deviations of the MADAGASCAR measurements, i.e $\pm 42 \%(2 \sigma)$.

masses. This quantification is possible for low bulk density objects with a homogeneous emission source and considering gamma multi-emitter radionuclides to quantify. The described stochastic method makes it possible to estimate the activity or mass PDF of a radionuclide to be quantified. Although the experimental data describe distributions with larger variances than traditional nuclear measurement uncertainties, PDF are obtained without a priori information about measured objects. The information supplied to the method comes from the acquisition spectrum or objective information sources as measurement distance, object volume, etc. Beyond estimating a mass of radionuclide, the method makes it possible to estimate the efficiency curve of the measurement scene. With this, it should be possible to use the proposed method to quantify mono gamma-emitter radionuclides. Concerning the positive bias on the estimated mass of plutonium, we have clearly identified it and are currently investigating potential causes.

The first difficulty of this study is that the MADAGASCAR data are measurements and not standards. Due to the difficulty in generating standard sources of plutonium, this MADAGASCAR database was used. The second difficulty was finding the right comparison tools. Indeed, the problem was to compare scalars (mass of MADAGASCAR plutonium and uncertainties) with estimated conditional PDFs. The four proposed studies allow a correct comparison of the measured and estimated data. Additional tools can be added.

Finally, the alpha value defined in Eq. 15 and set to $5 \%$, to simplify the calculations, slightly overestimates the uncertainty of the net extracted areas used. An automatic integration of these uncertainties within the code must be carried out to refine the calculations.

\section{REFERENCES}

[1] G. F. Knoll, "Germanium Gamma-Ray Detectors," Radiation detection and measurement, 4th ed., Ed. USA: Wiley, 2010, pp. 424-428

[2] N. Guillot, "Quantification gamma de radionucléides par modélisation équivalente," Ph.D. dissertation, Dept. Fond. Sciences., B. Pascal, C. Ferrand, France, 2015.

[3] MCNP6 User's Manual, vers. 1.0, LANL, May 2013.

[4] A. Lyoussi, "Spectrométrie gamma et X," in Détection de rayonnements et instrumentation nucléaire, EDP Sciences, 2010, pp. 156-164.

[5] N. Guillot, T. Vigineix and N. Saurel, "Method for quantifying the uncertainty associated at the gamma ray spectrum raw data extraction by deconvolution software," 2013 3rd International Conference on Advancements in Nuclear Instrumentation, Measurement Methods and their Applications (ANIMMA), Marseille, France, 2013, pp. 1-6, doi: 10.1109/ANIMMA.2013.6727895.

[6] Clement, A., Saurel, N., Perrin, G. 2018. "Stochastic approach for radionuclides quantification," in EPJ Web of Conferences 170, 06002 (2018). https://doi.org/10.1051/epjconf/201817006002.

[7] Carasco, C.,"Coupling gamma ray spectrometry and tomography in a Bayesian frame," in Nuclear Inst. and Methods in Physics Research, A 990 164985, https://doi.org/10.1016/j.nima.2020.164985, 2021.

[8] Laloy E., Rogiers B., Bielen A., Boden S.,"Bayesian inference of 1D activity profiles from segmented gamma scanning of a heterogeneous radioactive waste drum," in Arxiv preprint arXiv:2101.02112, A 990 164985, https://arxiv.org/abs/2101.02112, 2021.

[9] Génie2000, Customization tools manual. (2006). Canberra.

[10] INTERWINNER, v7.0. ITECH Instruments.

[11] C. P. Robert, "Introduction," in Le choix Bayésien, Springer, Ed. Paris, France, 2006, pp. 6-29.

[12] W.L. Dunn and J.K. Shultis, "Markov Chain Monte Carlo," in Exploring Monte Carlo Methods, Academic Press, 2012, pp. 132-156.

[13] W.K. Hastings, "Monte Carlo Sampling Methods Using Markov Chains and Their Applications", Biometrika, 1970, vol. 57, pp. 97-109.

[14] C. P. Robert and G. Casella, "Algorithmes de Metropolis-Hastings," in Méthodes de Monte-Carlo avec R, Springer, Ed. France, 2011, pp. 142165

[15] O. Roustant, D. Ginsbourger, et al., "DiceKriging, DiceOptim : Two R Packages for the Analysis of Computer Experiments by Kriging-Based Metamodeling and Optimization," Journal of Statistical Software, vol. 51, pp. 1-11, Oct. 2012.

[16] C. K. I. Williams, C. E. Rassmusen., "Gaussian processes for machine learning," in MIT Press, 2006.

[17] J. Sacks, W. Welch, T. Mitchell, and H. Wynn., "Design and analysis of computer experiments," in Statistical Science, vol. 4, pp. 409-435, 1989.

[18] R. Faivre, B. Iooss, et al., "Exploration par construction de métamodèles," Analyse de sensibilité et exploration de modèles, Quæ, Ed. France, 2013, pp. 159-173

[19] Park, Jeong-Soo., "Optimal Latin-hypercube designs for computer experiments," in Journal of Statistical Planning and Inference, vol. 39, pp. 95-111, 1994.

[20] R. Faivre, B. Iooss, et al., "Echantillonage en grande dimension," Analyse de sensibilité et exploration de modèles, Quæ, Ed. France, 2013, pp. 66-68

[21] Michael D. McKay, Richard J. Beckman and William J. Conover., "A Comparison of Three Methods for Selecting Values of Input Variables in the Analysis of Output From a Computer Code," in Technometrics, vol. 42, pp. 55-61, 2000.

[22] R: A Language and Environment for Statistical Computing. R Foundation for Statistical Computing, version 3.2.2. Available at https://www.Rproject.org/

[23] Python Software Foundation. Python Language Reference, version 3.7. Available at http://www.python.org

[24] ISO. Guidance for gamma spectrometry measurement of radioactive waste. ISO 19017. Decembre 2015. pp 2.36, A.4.6

[25] Reilly, D and Ensslin, N and Smith, H Jr and Kreiner, S., "Passive nondestructive assay of nuclear materials, Chap. 6", United States, 1991.

[26] COFRAC. French organism of accrediation. https://www.cofrac.fr/ 